1996

\title{
Spiral Wave Formation in Three-Dimensional Excitable Media
}

Takashi Amemiya

Sándor Kádár

Petteri Kettunen

Kenneth Showalter

Follow this and additional works at: https://researchrepository.wvu.edu/faculty_publications

\section{Digital Commons Citation}

Amemiya, Takashi; Kádár, Sándor; Kettunen, Petteri; and Showalter, Kenneth, "Spiral Wave Formation in Three-Dimensional Excitable Media" (1996). Faculty Scholarship. 341.

https://researchrepository.wvu.edu/faculty_publications/341 


\title{
Spiral Wave Formation in Three-Dimensional Excitable Media
}

\author{
Takashi Amemiya, ${ }^{*}$ Sándor Kádár, Petteri Kettunen, ${ }^{\dagger}$ and Kenneth Showalter ${ }^{\ddagger}$ \\ Department of Chemistry, West Virginia University, Morgantown, West Virginia 26506-6045
}

(Received 16 July 1996)

\begin{abstract}
A mechanism for the generation of spiral waves in three-dimensional excitable media is described. Experiments using the photosensitive Belousov-Zhabotinsky reaction show that circular or spiral waves appear in the wake of a propagating wave following a local perturbation in illumination intensity. The induced waves arise from three-dimensional scroll waves striking the upper boundary of the excitable medium. Spiral waves are formed over a range of illumination intensities and perturbation times which place the induced wave in the vulnerable region of the refractory wave back. [S0031-9007(96)01342-7]
\end{abstract}

PACS numbers: 82.20.Wt, 05.45.+b, 47.54.+r

Spiral waves are observed in a wide variety of living and nonliving excitable media, e.g., heart tissue [1], animal retina [2], Xenopus laevis oocytes [3], and oscillatory [4,5] and surface catalyzed [6] chemical reactions. Spiral waves in heart tissue have been studied extensively, since they are thought to be a precursor to life-threatening cardiac arrhythmias [7], and studies of biological and chemical excitable media have led to a detailed understanding of spiral wave dynamics [8,9]. Much less is known about the origin of spiral waves, especially their spontaneous appearance, although several mechanisms have been advanced [10-12]. In this Letter we describe a novel mechanism for spiral wave formation in three-dimensional excitable media. Studies of the photosensitive Belousov-Zhabotinsky (BZ) reaction $[13,14]$ demonstrate that spiral waves may form in the refractory tail of a wave that is perturbed by local changes in excitability in a process involving the formation of three-dimensional scroll waves [15].

It is well known that isotropic perturbations cannot give rise to spiral behavior in isotropic, homogeneous media. Spirals can be generated by nonisotropic perturbations, however, such as in cross field stimulation, where a wave is initiated to propagate into the refractory wake of a second, orthogonal wave $[1,10]$. Spirals similarly arise when waves are initiated in the vulnerable region of the refractory wave back of another wave [16-18]. Spiral behavior occurs spontaneously in inhomogeneous excitable media, where nonuniform excitability and refractory properties give rise to initiations of irregular wave segments $[11,19,20]$. Spirals also arise when high frequency waves impinge on impenetrable obstacles [12,21].

The most common technique for producing spirals in the laboratory (or in computer simulations) is to inhibit activity in a wave by lowering the local excitability (or by mechanically breaking the wave), thereby creating free ends around which spiral waves develop. The mechanism for spiral wave generation presented here involves increasing the local excitability transverse to a propagating wave in a three-dimensional medium. Our experiments utilized a thin layer of silica gel [22] with ruthenium(II)-tris-2,2'-bipyridyl catalyst distributed uniformly throughout [23]. The gel was placed into a petri dish and infused with catalyst-free BZ solution. The medium was then exposed (from below) to a constant flux of spatially homogeneous $350-500 \mathrm{~nm}$ light. When illuminated with light of this wavelength, the rutheniumBZ reaction becomes less excitable because $\mathrm{Br}^{-}$, an inhibitor in the reaction, is produced in a photochemical cycle $[14,23,24]$. Since the light is absorbed as it passes through the medium, the excitability is attenuated in the transverse direction. The system was perturbed by using an opaque mask to block the light, thereby changing the local excitability.

The perturbation was applied by placing the mask perpendicular to the front of a propagating wave [Figs. 1(a) and 1(b)]. After a brief period of time, a secondary wave developed in the wake of the primary wave, evolving into either a circular or spiral wave depending on the length of the perturbation and the level of illumination [Figs. 1(c) and 1(d)]. Multiple wave initiations were exhibited for long perturbation times, typically with two circular waves or a spiral wave within a circular wave [Fig. 1(e)]. In all cases, each secondary wave appeared at nearly the same place the primary wave was at the moment the mask was applied. As described below, the secondary waves arise from perturbation-induced, three-dimensional scroll waves [15].

Numerical simulations provide insights into the underlying three-dimensional mechanism of the secondary wave generation. Calculations were carried out using the Tyson-Fife scaling [25] of the Oregonator [26], modified to describe the photosensitive $\mathrm{BZ}$ reaction [27]:

$$
\begin{gathered}
\frac{\partial u}{\partial t}=\nabla^{2} u+(1 / \epsilon)\left(q w-u w+u-u^{2}\right), \\
\frac{\partial v}{\partial t}=u-v, \\
\frac{\partial w}{\partial t}=\nabla^{2} w+\left(1 / \epsilon^{\prime}\right)(\phi-q w-u w+f v),
\end{gathered}
$$

where $u, v$, and $w$ are the dimensionless concentrations of $\mathrm{HBrO}_{2}$, catalyst, and $\mathrm{Br}^{-}$, respectively, $\nabla^{2}=\partial^{2} / \partial x^{2}+$ 


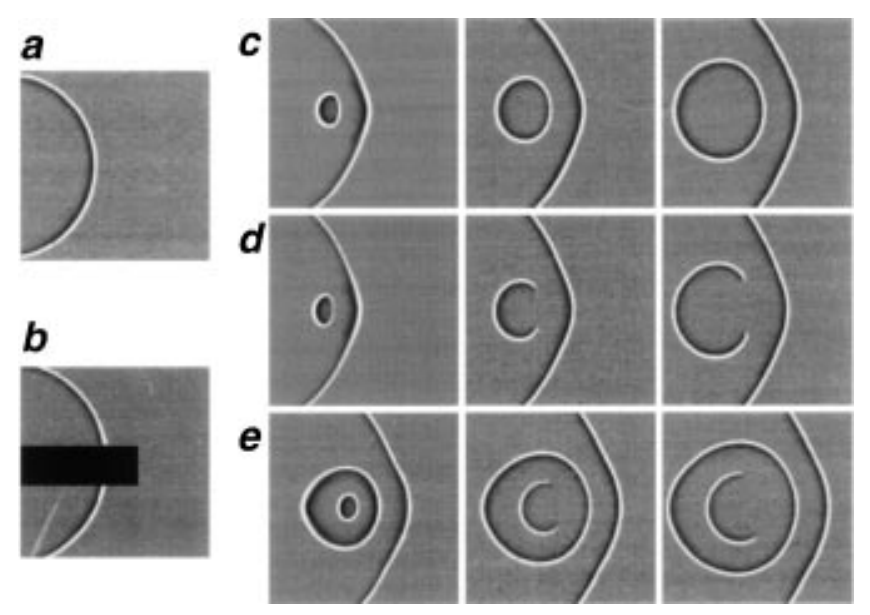

FIG. 1. Perturbation induced circular and spiral waves. White corresponds to the wave front and black to the wave back in the subtraction images ( $2 \mathrm{~s}$ delay). (a) Typical primary wave and (b) primary wave perturbed with $2 \times 8 \mathrm{~mm}$ opaque mask. (c) Circular wave formed with low illumination (output voltage of $3.5 \mathrm{~V}$ from photomultiplier tube, PMT) and short perturbation time (20 s). Images taken at 21, 35, and $65 \mathrm{~s}$ from the beginning of the perturbation. (d) Spiral wave formed with high illumination $(3.7 \mathrm{~V})$ and short perturbation time (20 s). Images taken at 21, 35, and $65 \mathrm{~s}$. (e) Spiral wave within a circular wave formed at high illumination $(3.7 \mathrm{~V})$ and long perturbation time $(40 \mathrm{~s})$. Images taken at 41,55 , and $65 \mathrm{~s}$. Image areas: $9.4 \mathrm{~mm} \times 9.4 \mathrm{~mm}$. Composition of the BZ solution (before bromination of malonic acid): $0.1 M \mathrm{NaBr}$, $0.25 M \mathrm{NaBrO}_{3}, 0.30 M$ malonic acid, and $0.49 M \mathrm{H}_{2} \mathrm{SO}_{4}$. Solutions were prepared with deoxygenated, doubly-distilled water and stored under argon gas. The silica gel medium $(20 \mathrm{~mm} \times 20 \mathrm{~mm} \times 0.5 \mathrm{~mm})$ was prepared by gelling a solution of $10 \%$ (w/w) $\mathrm{Na}_{2} \mathrm{SiO}_{3}$ and $1.0 \mathrm{~m} M$ ruthenium(II)-tris$2,2^{\prime}$-bipyridyl sulfate by acidifying with $\mathrm{H}_{2} \mathrm{SO}_{4}$. The primary wave was initiated with a silver wire at the left boundary of the gel. The covered petri dish was thermostated at $25.0 \pm 0.1{ }^{\circ} \mathrm{C}$ and flushed with a slow flow of argon gas. The gel was illuminated with light from a $1.0 \mathrm{~kW}$ tungsten-halogen lamp passing through an integrating sphere (with inhomogeneity less than $0.1 \%$ over the field) and a $350-500 \mathrm{~nm}$ bandpass filter. The light transmitted through the medium was monitored with a CCD video camera with a $460 \mathrm{~nm}$ narrow bandpass filter.

$\partial^{2} / \partial y^{2}+\partial^{2} / \partial z^{2}$ is the Laplacian operator, and $\phi$ gives the rate of bromide production from the irradiation. This rate is proportional to the light intensity [27], which varies in the $z$ direction according to a modified Beer-Lambert law:

$$
\phi(z)=\phi_{0} e^{-\alpha z}
$$

where $\alpha$ includes the molar absorption coefficient and concentration of the reduced catalyst, $\phi_{0}$ includes the quantum efficiency for the photochemical production of $\mathrm{Br}^{-}$, and $z$ is the dimensionless distance from the bottom surface of the medium. The calculation was simplified by treating $\alpha$ as a constant parameter, with the photoinduced bromide generation dependent only upon the value of $z$.

Successive images of the simulated three-dimensional behavior are shown in Fig. 2. The $(x, z)$ cross sections corresponding to each of the $(x, y)$ images show the primary wave only slightly penetrating the gel because of the inhibitory effect of the light [cf. Fig. 2(a)]. When the mask is applied [Fig. 2(b)], the wave penetrates into the medium to form a rotating scroll wave [Figs. 2(c), 2(d), and 2(e)]. The wave curls to strike the upper surface [Fig. 2(f)], giving rise to a wave that evolves into a circular or spiral wave [Figs. 2(g) and 2(h)], depending on the perturbation time and light intensity.

Experiments carried out to determine the effects of the perturbation time and light intensity on the development of the secondary waves are summarized in Fig. 3. The phase diagram shows that no secondary waves appear when the masking time is less than $\sim 15 \mathrm{~s}$, which, according to the behavior in the simulations, is about one-half the scroll-wave period. With increasing masking time, the secondary waves change from spiral to circular and then to multiple waves.

The secondary wave formation is limited to a narrow range of light intensity, as shown in Fig. 3. The upper limit of the range is determined by the complete inhibition of wave propagation due to the light-induced bromide production; the lower limit is defined by the failure of secondary wave formation. At lower light intensities, the primary wave penetrates deeper into the
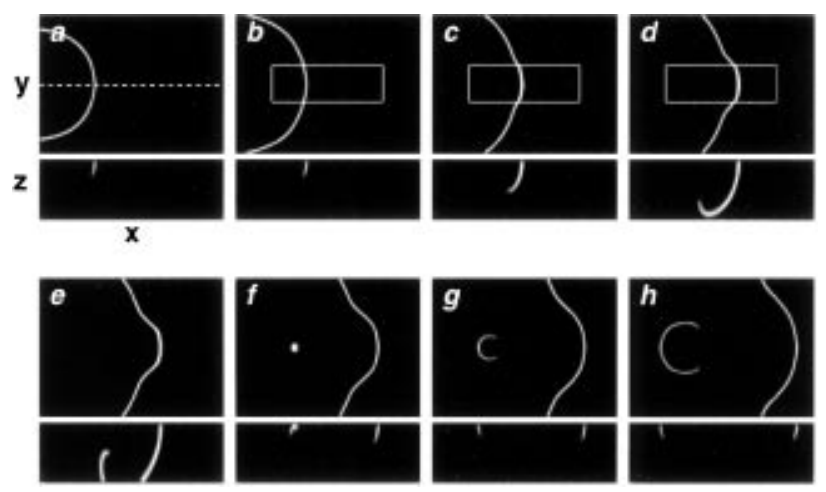

FIG. 2. Three-dimensional simulations of perturbationinduced spiral wave formation. Concentrations of $u$ are shown, with white assigned to the highest value in the wave front and black assigned to the steady-state value. Two images are shown for each sampling time: The upper $(x, y)$ image shows the top view as in Fig. 1; the lower $(x, z)$ image is the middle cross section [dashed line in $(x, y)$ image] along the direction of wave propagation. The $z$ direction is expanded twofold relative to the $x$ and $y$ directions. The white-outlined rectangle shows the mask, an area of $40 \times 60$ grid points with zero irradiation, which was imposed from $t=10.0$ to $t=16.3$. Parameters [28] of Eqs. (1) $-(3): \epsilon=6.61 \times 10^{-2}$, $\epsilon^{\prime}=3.67 \times 10^{-4}, q=9.52 \times 10^{-5}, f=2.05$. Parameters of Eq. (4): $\alpha=2.323 \times 10^{-1}$ and $\phi_{0}=2.00 \times 10^{-2}$. Sampling times: (a) 7.8, (b) 10.3, (c) 12.8, (d) 15.0, (e) 16.3, (f) 19.1, (g) 20.6, (h) 23.1. The finite difference form of Eqs. (1)-(3) was integrated in the three-dimensional rectangular grid $\left(N_{x} \times N_{y} \times N_{z}=200 \times 150 \times 32\right)$ by the explicit Euler method $(\Delta h=0.5, \Delta t=0.0125)$ with no-flux boundary conditions [29]. The behavior was unchanged when the step size was reduced by a factor of 2 . 


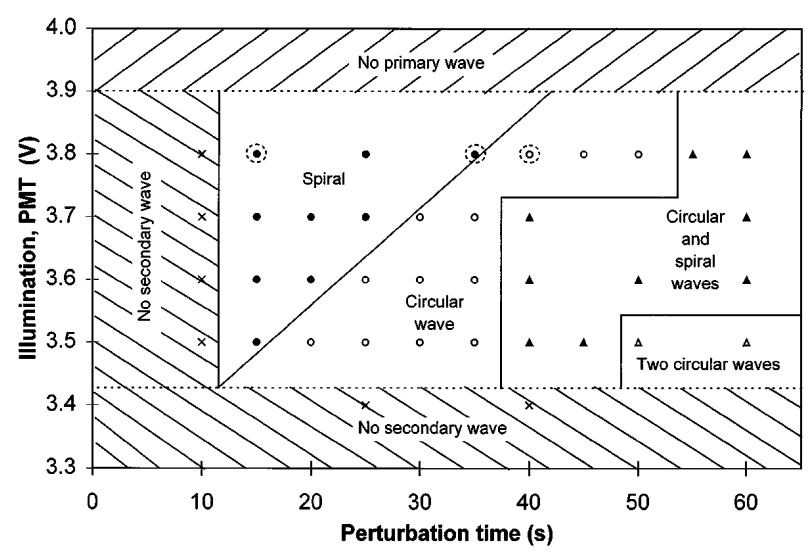

FIG. 3. Phase diagram showing behavior of the secondary waves as a function of the perturbation time and light intensity. Reactant concentrations and experimental conditions same as in Fig. 1. Dashed circles indicate internal reference points at which the light intensity was adjusted to maintain constant experimental conditions.

medium (in the $z$ direction) and a perturbation-induced scroll wave cannot rotate past the lower surface. Our experiments also showed that the gel thickness must be above a critical value (ca. $0.15 \mathrm{~mm}$ ) for secondary wave formation.

The subsequent evolution of the secondary waves into spiral or circular waves can be understood by considering the effects of the refractory wave back of the primary wave. At low light intensities there is less photoinduced bromide production [14,23,24], and the secondary wave is able to propagate in all directions, even against the increasing bromide gradient of the refractory wave back. At high light intensities, the higher bromide concentration adds to the refractory wave back sufficiently to partially inhibit the secondary wave in this region [16-18]. The result is a wave segment with two free ends, as shown in Fig. 1(d), which gives rise to counterrotating spiral waves.

The perturbation time plays a similar role in determining the features of the secondary waves. When the mask is removed before the appearance of the secondary wave, a spiral wave is formed in response to the photoinduced bromide production. When the perturbation time is longer, the extent of the local bromide production is reduced and circular waves are exhibited. If the mask is applied for more than 1.5 but less than 2 scroll-wave periods, a spiral wave forms within a circular secondary wave, where now the refractory tail of the initial secondary wave gives rise to the spiral behavior. For longer masking times, two circular waves form.

The mechanism for spiral wave generation described here relies on perturbations that locally change the character of the medium from effectively two dimensional to effectively three dimensional, permitting a scroll wave to develop. Perturbations of appropriate duration yield secondary wave formation in the vulnerable region of the preceding wave, with the subsequent development of counterrotating spiral waves. The process is not restricted to secondary waves arising from a "conditioning wave" but can occur with subsequent secondary waves. The spontaneous formation of spiral waves in excitable media such as heart tissue could occur by this mechanism if appropriate fluctuations of excitability take place in the thickness of the medium.

We thank Dr. Tomohiko Yamaguchi of the National Institute of Materials \& Chemical Research, Tsukuba, Japan, for discussions on photoinduced wave initiation. This research was supported by the National Science Foundation (CHE-9531515) and the Petroleum Research Fund (29565-AC6).

Note added. - A recent report by Yoneyama [30] describes a double-layered Belousov-Zhabotinsky system in which perturbation-induced scroll waves are exhibited.

*Permanent address: Department of Chemical Systems, National Institute of Materials \& Chemical Research, Tsukuba, Ibaraki 305, Japan.

${ }^{\dagger}$ Permanent address: University of Oulu, Department of Physical Sciences, Division of Biophysics, Linnanmaa, FIN-90570 Oulu, Finland.

\$To whom correspondence should be addressed.

[1] J. M. Davidenko, A. V. Pertsov, R. Salomonsz, W. Baxter, and J. Jalife, Nature (London) 355, 349-351 (1992).

[2] A. Gorelova and J. Bures, J. Neurobiol. 14, 353-363 (1983).

[3] J. Lechleiter, S. Girard, E. Peralta, and D. Clapham, Science 252, 123-126 (1991).

[4] K. J. Lee and H. L. Swinney, Phys. Rev. E 51, 1899-1951 (1995).

[5] A. T. Winfree, Science 175, 634-636 (1972).

[6] R. Imbihl and G. Ertl, Chem. Rev. 95, 697-733 (1995).

[7] A. T. Winfree, Science 270, 1224-1225 (1995).

[8] A.T. Winfree, When Time Breaks Down (Princeton University Press, Princeton, 1987).

[9] Chemical Waves and Patterns, edited by R. Kapral and K. Showalter (Kluwer, Dordrecht, 1995), Chaps. 1-6.

[10] A T. Winfree, J. Theor. Biol. 138, 353-405 (1989).

[11] G. K. Moe, W.C. Rheinboldt, and J. A. Abildskov, Am. Heart J. 67, 200-220 (1964).

[12] K. I. Agladze, J. P. Keener, S. C. Müller, and A. Panfilov, Science 264, 1746-1748 (1994).

[13] A. N. Zaikin and A.M. Zhabotinsky, Nature (London) 225, 535-537 (1970).

[14] L. Kuhnert, K. I. Agladze, and V.I. Krinsky, Nature (London) 337, 244-247 (1989).

[15] A. T. Winfree, Science 181, 937-939 (1973).

[16] A. T. Winfree, in Oscillations and Traveling Waves in Chemical Systems, edited by R. J. Field and M. Burger (Wiley, New York, 1985), pp. 441-472.

[17] M. Gómez-Gesteira, G. Fernández-García, A.P. Muñuzuri, V. Pérez-Muñuzuri, V.I. Krinsky, C.F. Starmer, and V. Pérez-Villar, Physica (Amsterdam) 76D, 359-368 (1994).

[18] R. R. Aliev and A. V. Panfilov, Phys. Rev. E 52, 22872293 (1995). 
[19] O. Steinbock, P. Kettunen, and K. Showalter, Science 269, 1857-1860 (1995).

[20] S. K. Scott, J. D. B. Smith, and B. W. Thompson, J. Chem. Soc. Faraday Trans. 92, 325-327 (1996).

[21] A. Babloyantz and J. A. Sepulchre, Physica (Amsterdam) 49D, 52-60 (1991).

[22] T. Yamaguchi, L. Kuhnert, Z. Nagy-Ungvarai, S. C. Müller, and B. Hess, J. Phys. Chem. 95, 5831-5837 (1991).

[23] O. Steinbock, V. Zykov, and S. C. Müller, Nature (London) 366, 322-324 (1993).

[24] P. K. Srivastava, Y. Mori, and I. Hanazaki, Chem. Phys. Lett. 190, 279-284 (1992).
[25] J. J. Tyson and P. C. Fife, J. Chem. Phys. 73, 2224-2237 (1980).

[26] R J. Field and R. M. Noyes, J. Chem. Phys. 60, 18771884 (1974).

[27] H. J. Krug, L. Pohlmann, and L. Kuhnert, J. Phys. Chem. 94, 4862-4866 (1990).

[28] R. J. Field and H.-D. Försterling, J. Phys. Chem. 90, 5400-5407 (1986).

[29] W. H. Press, S. A. Teukolsky, W. T. Vetterling, and B.P. Flannery, Numerical Recipes in $C$ (Cambridge University Press, Cambridge, England, 1992), 2nd ed.

[30] M. Yoneyama, Chem. Phys. Lett. 254, 191 (1996). 